\title{
Liver metastases from maxillary sinus sinonasal undifferentiated carcinoma: A case report
}

\author{
VITTORIO D'AGUANNO ${ }^{1}$, MASSIMO RALLI ${ }^{1}$, BRUNA CERBELLI ${ }^{2}$, \\ ANTONIO GRECO $^{1}$ and MARCO DE VINCENTIIS ${ }^{3}$ \\ Departments of ${ }^{1}$ Sense Organs, ${ }^{2}$ Radiological Oncological and Pathological Sciences, and \\ ${ }^{3}$ Oral and Maxillofacial Sciences, Sapienza University of Rome, I-00186 Rome, Italy
}

Received November 6, 2018; Accepted March 13, 2019

DOI: $10.3892 / \mathrm{ol} .2019 .10251$

\begin{abstract}
Sinonasal undifferentiated carcinoma (SNUC) is a rare malignancy arising from the nasal cavity with neuroendocrine differentiation and aggressive behavior. When first diagnosed, SNUC is often locally advanced with nodal and distant metastases. The present study indicates a rare case of sole metastases to the liver from maxillary sinus undifferentiated carcinoma. Diagnosis was confirmed by total body computed tomography and immunohistochemical analysis on tissues from both maxillary and liver neoplastic tissues. SNUC is an aggressive malignancy characterized by a poor prognosis. Distant metastases commonly involve bones and lungs and should always be suspected in patients with locally advanced stages. Distant metastases can be found in unexpected organs due to dissemination via collateral venous plexuses; isolated liver metastatic disease may also occur. Although SNUC is considered a chemo-sensitive malignancy, chemotherapy for metastatic SNUC needs to be further explored.
\end{abstract}

\section{Introduction}

Sinonasal undifferentiated carcinoma (SNUC) is a rare malignancy with neuroendocrine differentiation accounting for nearly $5 \%$ of all sinonasal malignancies (1). SNUC is a distinct clinicopathologic entity that must be distinguished from other sinonasal malignancies.

SNUC arises in the nasal cavity and is usually locally advanced when first diagnosed (2). Presenting symptoms depend on the primary site and often include epistaxis, bloody rhinorrhea, visual changes, nasal obstruction, headache, and facial pain (3).

Correspondence to: Dr Massimo Ralli, Department of Sense Organs, Sapienza University of Rome, Viale del Policlinico 155, I-00186 Rome, Italy

E-mail: massimo.ralli@uniroma1.it

Key words: sinonasal undifferentiated carcinoma, metastatic cancer, liver metastases, chemotherapy
Clinically positive regional lymph nodes are present at diagnosis in 10 to $30 \%$ of patients (4). Although distant metastases are uncommon at presentation, their occurrence has been often reported mainly involving the lungs and bones (2). The presence of a sole metastasis to the liver at diagnosis in a patient with SNUC is unusual and only a few cases have been reported in the literature. In this article, we report the case of a 50-year-old woman presenting with nasal obstruction and diagnosed with a liver metastasis from SNUC.

\section{Case report}

A 50-year-old women presented to the Otolaryngology Department of our University reporting a three-month progressively worsening history of left maxillary swelling with mild facial pain and left nasal obstruction. The patient denied recent history of epistaxis, abdominal pain or previous virus-related hepatitis. No alcohol consumption or smoking were reported.

Otolaryngologic examination showed a painful, tough swelling in the left maxillary region. No periorbital proptosis, cranial nerve palsy or paresthesia were found. Neck examination showed the presence of palpable nodes in the submandibular level adherent to the underlying structures. Fiberoptic examination showed a reddish mass in the middle meatus, arising from the medial wall of the maxillary sinus; no signs of recent bleeding were observed.

A total body computed tomography (CT) scan with iodine contrast was performed. The exam showed a soft tissue mass in the left maxillary sinus measuring $4.6 \times 4.1 \mathrm{~cm}$ and extending to the nasal cavity, with signs of bone erosion in the medial and anterior wall of the maxillary sinus. Contrast enhancement was uniformly distributed in the neoplastic tissue (Fig. 1). Necrotic lymph nodes were present in the left submandibular angle near the jugular digastric region. Abdominal CT scan showed multiple liver expansive lesions localized in the right lobe (Fig. 2). No CT features of liver cirrhosis or hepatocellular carcinoma were present.

$\mathrm{A}^{18} \mathrm{~F}-\mathrm{FDG}$ PET/CT scan was performed to exclude the presence of further distant metastasis and no additional localization of the disease were observed (Fig. 3).

After informing the patient, biopsies of both nasal and liver tissues were performed. Biopsy of the left maxillary sinus showed massive infiltration of undifferentiated epithelial 


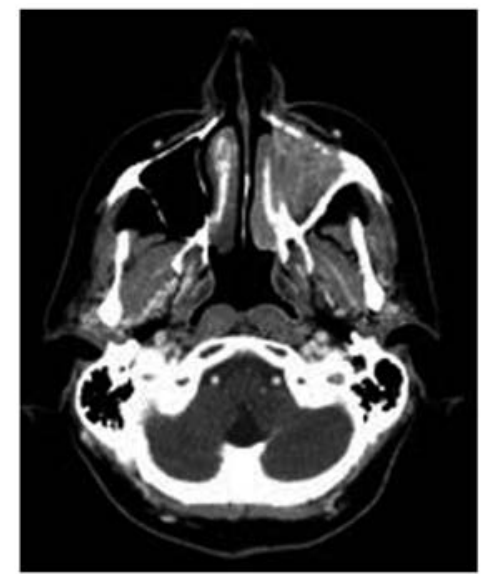

Figure 1. Axial contrast-enhanced computed tomography scan of the head showing a soft tissue mass measuring $4.6 \times 4.1 \mathrm{~cm}$ in the left maxillary sinus. Signs of bone erosion are evident in the medial and anterior walls of the maxillary sinus, with mass extension into the nasal cavity.

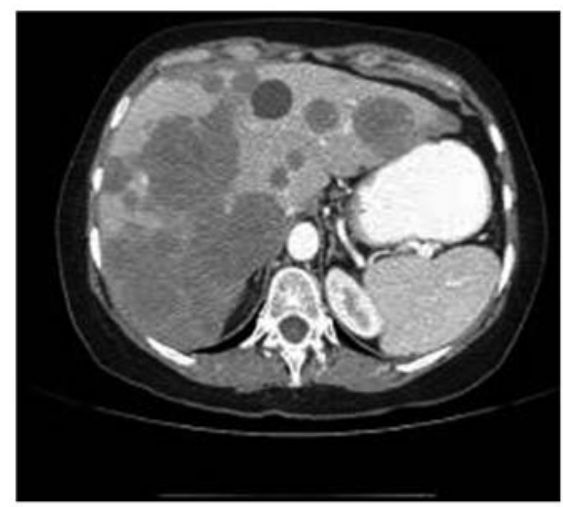

Figure 2. Venous-phase computed tomography scan showing multiple, bilobar liver lesions. Ultrasound-guided biopsy of the largest lesion was consistent with metastasis of sinonasal undifferentiated carcinoma.
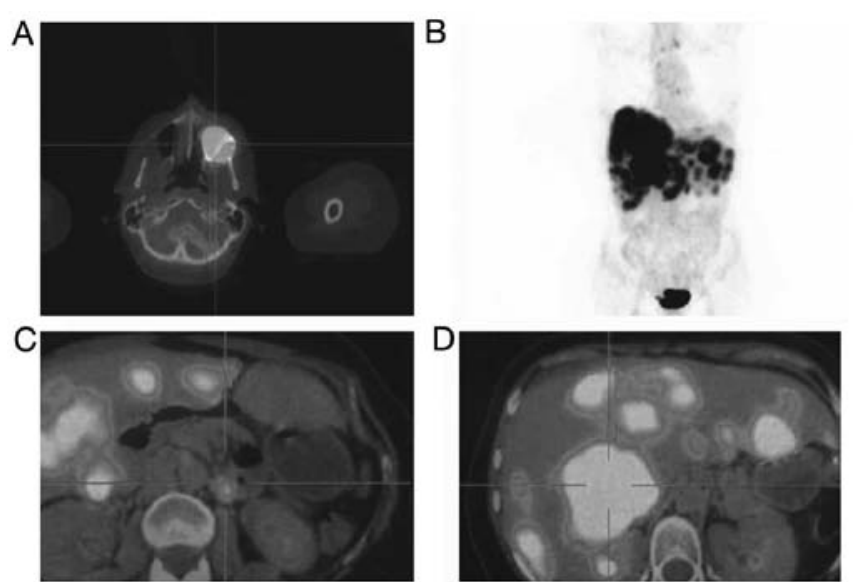

Figure 3. (A-C) ${ }^{18}$ F-FDG PET/CT showing a severe uptake of FDG in the maxillary sinus and in the liver. (D) The fusion image of 18-FDG-PET/CT in the coronal plane indicates no further uptake. ${ }^{18} \mathrm{~F}$-labeled fluoro-2deoxyglucose-positron emission tomography/computed tomography.

neoplastic cells, with scarce cytoplasm and enlarged nucleus, organized in nests of cells and surrounded by a fibrous stroma. High mitotic activity without necrotic areas was present.
Immunohistochemical assay confirmed the diagnosis of undifferentiated maxillary sinus carcinoma (Fig. 4).

Ultrasound-guided percutaneous liver biopsy of the largest lesion revealed nests of cells with irregular margins and slightly eosinophilic cytoplasm, with enlarged nuclei and basophilic inclusions. Immunohistochemical analysis revealed positivity for CK MNF 116, CK7 CK8 NSE and CEA. Proliferation cell index evaluated through Ki-67 was nearly $60 \%$. No signs of cirrhosis, hepatocellular carcinoma features and additional masses in the surrounding hepatic parenchyma were found (Fig. 5). According to these findings, a diagnosis of metastatic SNUC was made.

After multidisciplinary discussion of the case, surgery was excluded due to presence of liver metastases, and induction chemotherapy followed by a chemo-radio therapy was proposed. The patient underwent 6 cycles of chemotherapy with carboplatin plus taxol before radiation therapy (2 Gy/die-33 cycles). Although induction chemotherapy was able to reduce both nasal and liver masses, the patient died shortly after.

\section{Discussion}

SNUC is a rare malignancy first described by Frierson et al (5) in 1986. The etiology of SNUC is unknown; however, it is believed that the tumor arises from malignant epithelial cells in the nasal mucosa (5). SNUC occurs most predominantly in males, suggesting etiological involvement of sexual hormones, smoking or occupational hazards (6).

The classification of this condition as a specific identity is still debated and nomenclature is often ambiguous. Thus, the application of a uniform classification system for neuroendocrine carcinoma of the head and neck is required (7). Nowadays, SNUC is considered to be part of the spectrum of neuroendocrine carcinomas including esthesioneuroblastoma, neurondocrine carcinoma, and small cell carcinoma (8). While esthesioneuroblastoma is considered a neuroectodermal malignancy, sinonasal neuroendocrine carcinoma (SNC) must be considered an epithelial malignancy and should be classified based on differentiation grade into well, moderately and poorly differentiated. Moreover, poorly differentiated SNC are further subdivided into a small and large cell variant (4). Thus, histological diagnosis of these malignancies always requires carefully assessment of the specimen by an expert pathologist.

The prognosis of SNUC is better than small cell carcinoma, similar to neuroendocrine carcinoma, and worse than esthesioneuroblastoma (8). Prognosis in influenced by the lack of specific symptoms leading to delayed diagnosis (4); nearly $75 \%$ of SNUC patients present with a stage IV disease.

SNUC shows higher propensity to nodal and distant metastasis compared to other sinonasal malignancies (9). metastases can be found in unexpected organs due to dissemination via collateral venous plexuses. The presence of metastasis in the liver from the sinonasal region without further localizations, as reported in this case, is quite uncommon. In fact, the hematogenous spread of metastases to the liver would typically require passage through the lungs before entering the liver via the hepatic artery; therefore the exclusion of other metastases is essential (10). The absence of lung metastases, as in the present case, could be explained by dissemination 
A

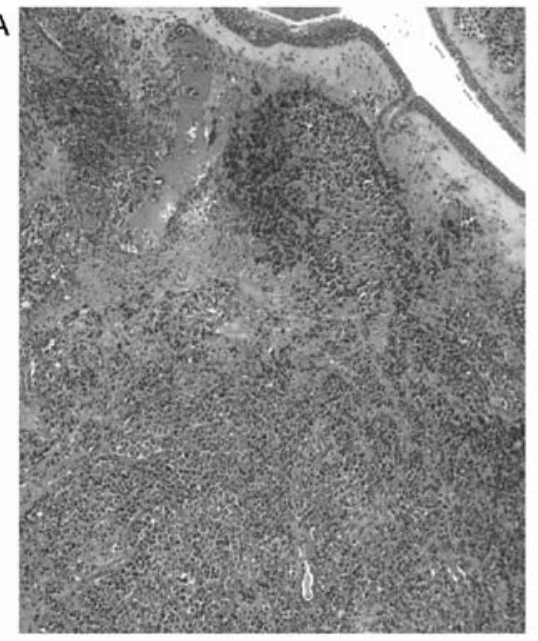

$\mathrm{B}$
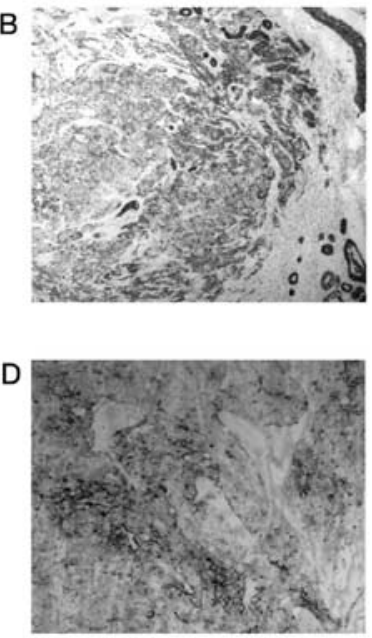
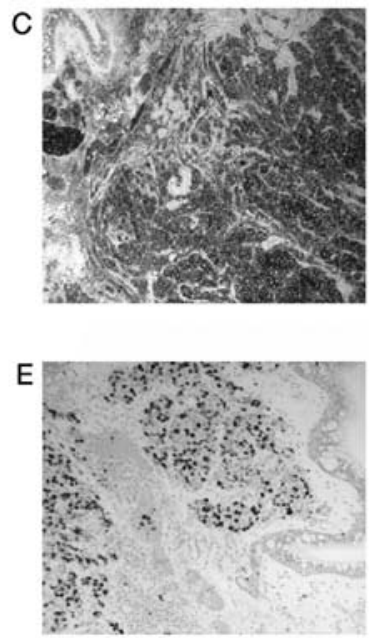

Figure 4. (A) Sinonasal biopsy showing an invasive tumour with a diffuse pattern growth (original magnification, x5). (B and C) Neoplastic cells are diffusely positive for mouse monoclonal cytokeratin antibody MNF 116 and neuron specific enolase staining (original magnification, x5). (D) Neoplastic cells are focally positive for CEA staining (original magnification, x10). (E) High proliferative index was also reported (Ki67 immunostaining, original magnification, x10).

A

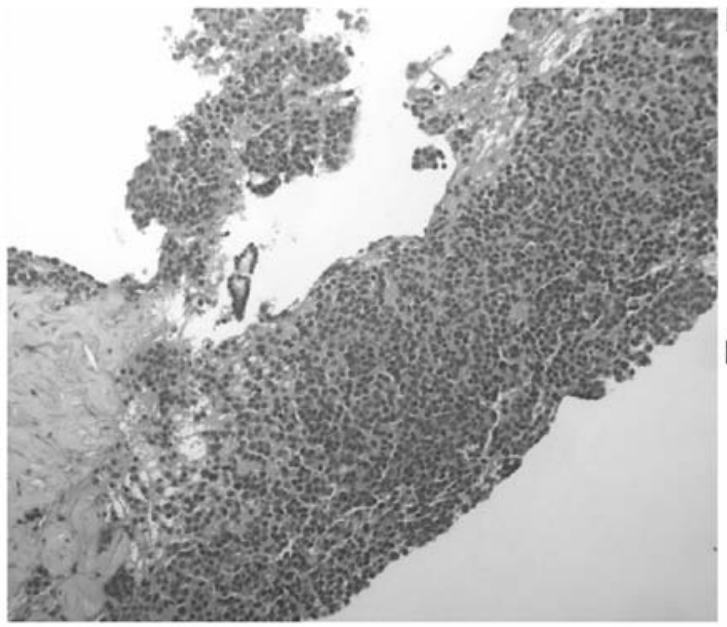

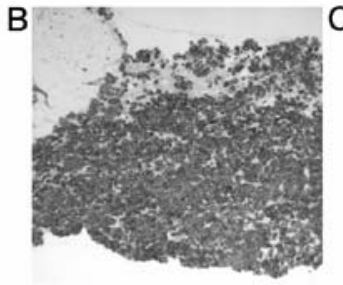
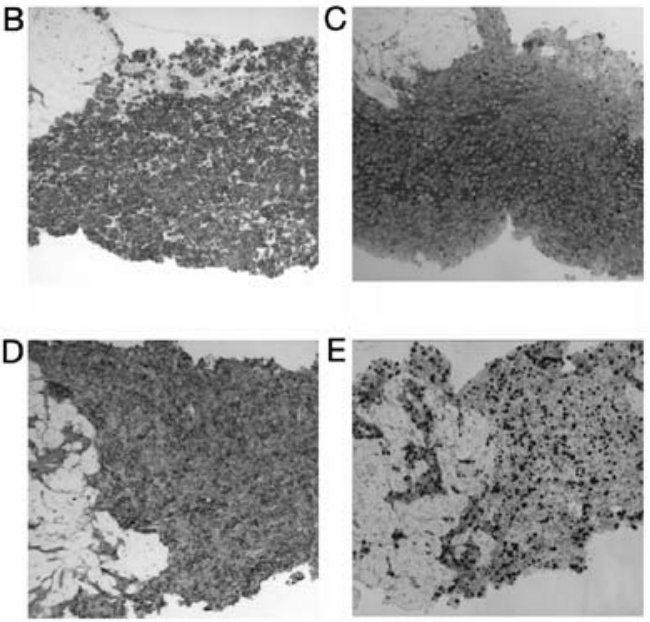

Figure 5. (A) Liver biopsy showing a neoplasia growing in a diffuse pattern growth (hematoxylin and eosin; original magnification, x5). (B-D) Neoplastic cells are diffusely positive for mouse monoclonal cytokeratin antibody MNF 116, carcinoembryonic antigen and neuron specific enolase staining (original magnification, x10). (E) High proliferative index was also recorded (KI67 immunostaining; original magnification, x10).

via the vertebral venous plexus and its numerous anastomoses with the azygos veins, which constitute a longitudinal network parallel to the inferior vena cava (10). In this case, tumor cells may have reached the liver via the portal system through the azygos and peri-oesophageal veins although other collateral pathways cannot be excluded.

A recent paper reported a case of a patient with metastatic SNUC with a single metastasis in the liver. The authors reported surgical resection of the liver followed by an orthotopic liver transplantation. The patient underwent a post-transplantation immuno-suppressive regimen followed by systemic adjuvant chemotherapy. However, the follow-up period was limited to 13 months and the chemotherapy regimen was not reported (11).

Another case of solitary liver metastasis from an ethmoid sinus adenocarcinoma has been reported. The patient was treated with endonasal tumor resection, followed by external beam radiotherapy with a dose fractionation of $60 \mathrm{~Gy}$ in 30 fractions over 6 weeks. After about 3 years of follow-up, a single liver metastasis of intestinal-type adenocarcinoma was detected and treated by partial hepatectomy without adjuvant therapy (10).

Due to the common delayed diagnosis, treatment of SNUC is multimodal. For locally advanced stages, open craniofacial resection has been proposed as the main treatment for a long time (6), although the introduction of endoscopic sinus and skull base surgery has modified the surgical options.

Radiotherapy should always be considered as part of treatment in the postoperative setting. Moreover, the addition of chemotherapy to radiotherapy seems to provide a survival advantage, although evidence is limited and still controversial (6). In fact, although SNUC is considered a chemo-sensitive disease, data about responses to induction chemotherapy are limited. The use of induction chemotherapy may improve both locoregional control and decrease distant metastases (12) and provides a rationale for the use of both induction and concurrent chemotherapy.

The role of chemotherapy in metastatic SNUC has not been clearly established yet (10). The administration of a 
platinum-base chemotherapy concurrent with radiation has been demonstrated to improve locoregional control and survival and has been the mainstream of treatment in many institutions for metastatic SNUC (12). Moreover, platinum-base chemotherapy had been associated with additional drugs, such as etoposide and taxol, to improve local control.

In conclusion, SNUC is a rare malignancy with neuroendocrine differentiation. Since the tumor arises in the nasal cavity, patients generally present nonspecific symptoms such as nasal obstruction and epistaxis, thus SNUC is often locally advanced or metastatic when first diagnosed. Metastatic SNUC should always be suspected in patients with locally advanced SNUC; metastases can be found in unexpected organs due to dissemination via collateral venous plexuses.

\section{Acknowledgements}

Not applicable.

\section{Funding}

No funding was received.

\section{Availability of data and materials}

The datasets used and analysed during the current study are available from the corresponding author on reasonable request.

\section{Authors' contributions}

VD and MR wrote the manuscript. VD, MR, AG and MDV contributed to the treatment of the case. BC reviewed histological specimens. All authors read and approved the manuscript.

\section{Ethics approval and consent to participate}

Written informed consent for the publication of any associated data and accompanying images was obtained from the patients or their guardians.

\section{Patient consent for publication}

The patient provided written informed consent for the publication of their data in this study.

\section{Competing interests}

The authors declare that they have no competing interests.

\section{References}

1. Mitchell EH, Diaz A, Yilmaz T, Roberts D, Levine N, DeMonte F, Hanna EY and Kupferman ME: Multimodality treatment for sinonasal neuroendocrine carcinoma. Head Neck 34: 1372-1376, 2012.

2. Mendenhall WM, Mendenhall CM, Riggs CE Jr, Villaret DB and Mendenhall NP: Sinonasal undifferentiated carcinoma. Am J Clin Oncol 29: 27-31, 2006.

3. Musy PY, Reibel JF and Levine PA: Sinonasal undifferentiated carcinoma: The search for a better outcome. Laryngoscope 112: 1450-1455, 2002

4. van der Laan TP, Iepsma R, Witjes MJ, van der Laan BF, Plaat BE and Halmos GB: Meta-analysis of 701 published cases of sinonasal neuroendocrine carcinoma: The importance of differentiation grade in determining treatment strategy. Oral Oncol 63: 1-9, 2016.

5. Frierson HF Jr, Mills SE, Fechner RE, Taxy JB and Levine PA: Sinonasal undifferentiated carcinoma. An aggressive neoplasm derived from schneiderian epithelium and distinct from olfactory neuroblastoma. Am J Surg Pathol 10: 771-779, 1986.

6. Morand GB, Anderegg N, Vital D, Ikenberg K, Huber GF, Soyka MB, Egger M and Holzmann D: Outcome by treatment modality in sinonasal undifferentiated carcinoma (SNUC): A case-series, systematic review and meta-analysis. Oral Oncol 75: 8-34, 2017.

7. van der Laan TP, Bij HP, van Hemel BM, Plaat BE, Wedman J, van der Laan BF and Halmos GB: The importance of multimodality therapy in the treatment of sinonasal neuroendocrine carcinoma. Eur Arch Otorhinolaryngol 270: 2565-2568, 2013.

8. Rosenthal DI, Barker JL Jr, El-Naggar AK, Glisson BS, Kies MS, Diaz EM Jr, Clayman GL, Demonte F, Selek U, Morrison WH, et al: Sinonasal malignancies with neuroendocrine differentiation: Patterns of failure according to histologic phenotype. Cancer 101: 2567-2573, 2004.

9. Castelnuovo P, Turri-Zanoni M, Battaglia P, Antognoni P, Bossi P and Locatelli D: Sinonasal malignancies of anterior skull base: Histology-driven treatment strategies. Otolaryngol Clin North Am 49: 183-200, 2016.

10. Caselhos S, Ferreira C, Jácome M and Monteiro E: Liver metastasis of ethmoid sinus adenocarcinoma. Eur Ann Otorhinolaryngol Head Neck Dis 132: 157-159, 2015.

11. De Simone P, Coletti L, Campani D, Falcone A and Filipponi F: Liver transplantation for metastatic sinonasal undifferentiated carcinoma: A case report. Transplant Proc 40: 3821-3822, 2008.

12. Rischin D, Porceddu S, Peters L, Martin J, Corry J and Weih L: Promising results with chemoradiation in patients with sinonasal undifferentiated carcinoma. Head Neck 26: 435-441, 2004. 